\title{
Metodología de ordenamiento territorial en la RACCN, un aporte al desarrollo con identidad ${ }^{1}$
}

\author{
Methodology of territorial ordering in RACCN, a contribution to the development with identity
}

\author{
Keith Sankara Narváez Ismael² \\ Elizabeth Salomon McCLean ${ }^{3}$ \\ Enrique Cordón Suárez ${ }^{4}$
}

\section{Resumen}

Con el fin de proponer una metodología, que responda a la nueva realidad de distribución territorial de la Región Autónoma de la Costa Caribe Norte de Nicaragua, se presenta la "Metodologías de ordenamiento territorial en la RACCN, un aporte al desarrollo con identidad". Tuvo como caso de estudio el territorio indígena miskitu de Twi Waupasa y el territorio Mayangna Sauni Bas. La investigación metodológicamente fue desde el paradigma de la ciencia crítica, la cual busca comprender la realidad, para su posterior transformación. Se dinamizó la información de campo de los grupos metas, así también los procesos que se ha desarrollado en los planes de ordenamientos territoriales en el ámbito nacional, regional y territorial. Teniendo como base las entrevistas directas, grupo focales, revisión de investigaciones primarias, así como la revisión de bibliografías secundarias que sostiene las teorías propuestas en la investigación. Los estudios de Ordenamiento y Desarrollo Territorial nacional, tienen un enfoque departamental y municipal, y su metodología está comprendida en cinco Etapas en base al INETER, y siete momentos metodológicos en base al Nuevo FISE-GIZ, que definió una metodología específica para la RACCN. La propuesta metodologica tiene 5 momentos estratégicos (Consentimiento previo libre e informado, Diagnóstico participativo, Prospectiva y modelo futuro, Presentación, Concertación y aprobación e Instrumentación e Implementación) con sus 41 acciones. Esta propuesta aportará de manera significativa para los territorios indígenas y comunidades de la RACCN, en la gestión, articulación, desarrollo, incidencia, gobernabilidad, gobernanza y así responder al paradigma del buen vivir y vivir bien de los pueblos indígenas de la Costa Caribe nicaragüense.

Palabras clave: Metodología; Planificación; Ordenamiento Territorial.

\section{Abstract}

In order to propose a methodology that responds to the new reality of territorial distribution of the Autonomous Region of the North Caribbean Coast of Nicaragua, the "Methodologies of territorial organization in RACCN, a contribution to the development with identity" is presented. This investigation had the Twi Waupasa Miskitu indigenous territory and the Mayangna Sauni Bas territory as a case study. The methodological research was from the paradigm of critical science, which seeks to understand reality, for further transformation. The field information of the target groups was dynamized, as well as the

\footnotetext{
1 XIV Congreso Latinoamericano y del Caribe de Extensión Universitaria y Segundo Congreso Centroamericano de Compromiso Social para la vinculación social de la universidad con la sociedad.

2 Máster en Planificación y Desarrollo Regional con Identidad, Coordinador Cooperación Externa de la Universidad de las Regiones Autónomas de la Costa Caribe Nicaragüense - Recinto Universitario Bilwi. Correo: cooperacion.bilwi@uraccan.edu.ni ORCID: https://orcid.org/0000-0003-3268-2643

3 Máster en Planificación y Desarrollo Regional con Identidad. Coordinadora Instituto de Promoción e Investigación Lingüística y Revitalización Cultural de la Universidad de las Regiones Autónomas de la Costa Caribe Nicaragüense - Recinto Universitario Bilwi. Correo: elizabeth.salomon@gmail.com

4 Doctor en Agroecología Tropical. Vicerrector de la Universidad de las Regiones Autónomas de la Costa Caribe Nicaragüense-Recinto Universitario Bilwi. Correo: vicerrectoria.bilwi@uraccan.edu.ni
}

Recibido: 17/01/2018 - Aprobado: 05/04/2018 
processes that have been developed in the territorial ordinances plans at national, regional and territorial spheres. The research was based on direct interviews, focus group, review of primary research, as well as the review of secondary bibliographies that support the theories proposed in the study. The studies of national Territorial Planning and Development have a departmental and municipal approach, and its methodology is comprised of five Stages based on the INETER, and seven methodological moments based on the New FISE-GIZ, which defined a specific methodology for the RACCN. The methodological proposal has 5 strategic moments (Prior, Free and Informed Consent Letter, participatory diagnosis, prospective and future model, presentation, agreement and approval and instrumentation and implementation) with its 41 actions. This proposal will contribute significantly to the indigenous territories and communities from RACCN, in the management, articulation, development, advocacy, governance, and thus respond to the paradigm of good living and live well of the indigenous peoples of the Nicaraguan Caribbean Coast.

Key words: Methodology, Planning, Territory Ordering.

\section{Introducción}

El proceso de demarcación y titulación de las tierras indígenas y afrodescendientes es un hecho sin precedentes en la Costa Caribe de Nicaragua. El Gobierno de Nicaragua ha titulado 22,452.27 $\mathrm{Km}^{2}$ del territorio nacional y casi un 75\% del territorio de la RACCN, Narvaez, K. (2014), a favor de las comunidades indígenas y étnicas, bajo las modalidades de tierras comunales y territoriales. Significa que a quinientos años de historia colonial y republicana, por fin las comunidades y pueblos originarios están gozando del reconocimiento institucional de los derechos que siempre han tenido sobre sus tierras y recursos.

Teniendo presente lo señalado, nos preguntamos ¿cómo están los planes de ordenamiento territorial a nivel de los territorios indígenas? Esta pregunta nos lleva a ver que existen planes de ordenamiento territorial que se han formulado y llevan su acción hasta el nivel de los municipios. Son propuestas metodológicas planteadas por INETER con un enfoque exógeno y no son pertinentes ni aplicables a las realidades de los territorios indígenas de la Costa Caribe nicaragüense.

En este sentido, se hace necesario crear y construir desde la base (comunidades), una verdadera metodología de planificación de uso de los recursos naturales, que trastoque la armonización con los conocimientos y costumbres ancestrales. Se busca el manejo y cuido de los recursos florísticos y faunístico, bajo una verdadera planificación de desarrollo con identidad comunal, territorial, regional en el marco del concepto del buen vivir; sin obviar lo existente en la temática de planificación que tiene Nicaragua y la RACCN.

Esta investigación presenta una propuesta metodológica de ordenamiento territorial con identidad, con un enfoque de respuesta a la nueva realidad de territorios indígenas de la RACCN en Nicaragua. Tiene su punto de partida en el análisis de metodologías utilizadas para elaborar planes de ordenamiento planteadas por el INETER y GIZ.

\section{Referencia de literatura}

\subsection{Comunidades indígenas y sus dinámicas}

Brenes (2007), plantea que una comunidad es un tipo de organización social cuyos miembros se unen para participar de objetivos comunes, donde algunos o muchos de los objetivos individuales se comparten o se identifican con los intereses colectivos. A mayor número de intereses compartidos habrá mayor cohesión social.

\subsection{El territorio}

Según Mendoza-Lewis (2009), el territorio se define como "un espacio geográfico, donde se dan relaciones entre los seres humanos y entre la naturaleza". 
De igual manera el Fondo Indígena (2008:40), plantea "el territorio es ante todo un espacio que presenta retos y oportunidades que va impulsando el conocimiento colectivo del pueblo que lo habita, que, a su vez, va reconstruyendo material, se trata de una apropiación cultural, social, económica, ecológica y política".

Para Larzon y Soto (2012:8), el territorio "es un espacio geográfico, pero es mucho más que eso, un territorio representa una jurisdicción, protegida en cierta medida por la ley (según cada país), en el que se pueden ejercer legalmente las normas tradicionales, la reproducción cultural y el autogobierno".

Según la ley 445, define territorio como el espacio geográfico que cubre la totalidad del hábitat de un grupo de comunidades indígenas o étnicas que conforman una unidad territorial donde se desarrollan, de acuerdo a sus costumbres y tradiciones (Art. 3).

\subsection{El ordenamiento territorial}

Según Zúñiga (2008:50) dice que ordenar: "es una acción administrativa del estado, que tiene que ver fundamentalmente con el uso y la ocupación del territorio

Camaño (2008:70) define que ordenamiento territorial: “...es el proceso de planeación, evaluación y control, dirigido a identificar y programar actividades humanas compatibles con el uso y manejo de los recursos naturales en el territorio nacional respetando la capacidad de carga.

Masirris A (2002), que la ordenación del territorio es: "la expresión espacial de la política económica, social, cultural y ecológica de toda sociedad"; y "a la vez una disciplina científica, una técnica administrativa y una política".

\subsection{Los procesos de ordenamiento territorial}

De acuerdo a Romero. R y Lorito. A (INETER, 2007) señalan que el proceso metodológico establece en las siguientes fases: La preparación, diagnóstico, prospectivo, la propuesta, instrumentación.

Quiñonero J. et al (2007), plantea que este modelo se ha utilizado como base para elaborar esta propuesta destinada al PRONOT, tras su correspondiente adaptación a la realidad nicaragüense en cuanto se refiere a sus características específicas y particulares. Según tal esquema, a cada unidad territorial: país, región, departamento, municipio, se aplica el tipo de figura legal de planificación territorial correspondiente a su nivel.

En este caso señala Masirris A (2002), que la débil visión prospectiva es otro de los rasgos comunes de las políticas latinoamericanas de ordenación del territorio. Su análisis se realiza a partir de dos indicadores: la formulación de objetivos a largo plazo y la incorporación en el proceso de toma de decisiones de criterios y métodos provenientes de la prospectiva, especialmente visiones alternativas de futuros deseados, escenarios alternativos de futuros posibles.

\subsection{Implementación de los planes de ordenamientos territoriales}

De acuerdo al PRODT, RACCN (Zúniga H. y Lippert T. 2008), la implementación de los planes de ordenamiento y desarrollo regional, se debe de revisar dos procesos que son importantes en la implementación de ella, la instrumentación y la operación. La instrumentación: Consiste en la creación de diferentes medios y herramientas, que garantizan la aplicación de manera efectiva de las declaraciones del nuevo PRODT. La operación: La operación consiste en la ejecución de las acciones propuestas por el PRODT, con el fin de establecer los escenarios definidos. 


\subsection{Metodología}

Según Sierra P. (2012), proviene de los vocablos griego método y logos que significan: estudio de los métodos. "Rama de la lógica que se encarga del estudio de los diferentes métodos para llegar al conocimiento crítico y reflexivos que permita la fundamentación de la ciencia”.

La metodología tiene su origen en el idioma griego, y se refiere al modelo aplicable que deben necesariamente seguir los métodos de investigación, aun cuando resulten cuestionables. También es la metodología es la ciencia que estudia aquellos métodos, sin detenerse en la validez pragmática sino justamente en la metodológica.

\subsection{Participación social}

En la mayoría de los casos, la participación social sólo tiene un carácter consultivo y, por tanto, no incide significativamente en las decisiones finales. Normalmente consiste en usar a unos pocos ciudadanos para tratar de mostrar un plan consensuado y, por lo tanto, legítimo socialmente (...) es notable en los países latinoamericanos, la ausencia de una cultura de la planificación participante y el escaso dominio de metodologías de consensos y de resolución de conflictos en los procesos de concertación, que permitan, por ejemplo, evitar que los actores poderosos impongan sus intereses individuales por encima del interés colectivo. (Massiris, 2001).

\subsection{Desarrollo con Identidad}

Para Hooker A. y Zapata Y. (2012), implica el impulso de acciones para el empoderamiento y la autogestión de los pueblos en el marco del desarrollo de sus propios procesos de transformación para el bienestar colectivo e individual.

\section{Metodología y materiales}

El estudio es prospectivo al basarse en la revisión y formulación de un proceso temporal, así también se asienta en un enfoque cualitativo, que se han desarrollado a nivel de la RACCN en los procesos de ordenamiento territorial. Se fundamenta y se formula con la participación de dos pueblos indígenas con diferentes culturas, ideologías, cosmovisiones, contextos geoeconómicos y políticos. Así también la participación de instancias endógenas y exógenas a la región autónoma que han desarrollado acciones para los procesos de ordenamiento territorial a nivel regional y local. La investigación tuvo su paradigma de la ciencia crítica, la cual busca comprender la realidad, para su posterior transformación.

El área de estudio es en la RACCN, teniendo punto de referencia el Territorio indígena de Twi Waupasa y el Territorio indígena de Mayangna Saunib. Territorios que cuentan con sus planes de ordenamiento y desarrollo territorial vigente y aprobado por las asambleas territoriales y avaladas por el gobierno regional como instrumento de planificación de sus territorios. Territorios, con diferentes administraciones municipales, una perteneciente al municipio de Siuna y la otra al municipio de Puerto Cabezas, que se vuelve significativo al momento de analizar geopolíticamente. Dos territorios con diferente etnia, cultura, tradición y cosmovisión. 


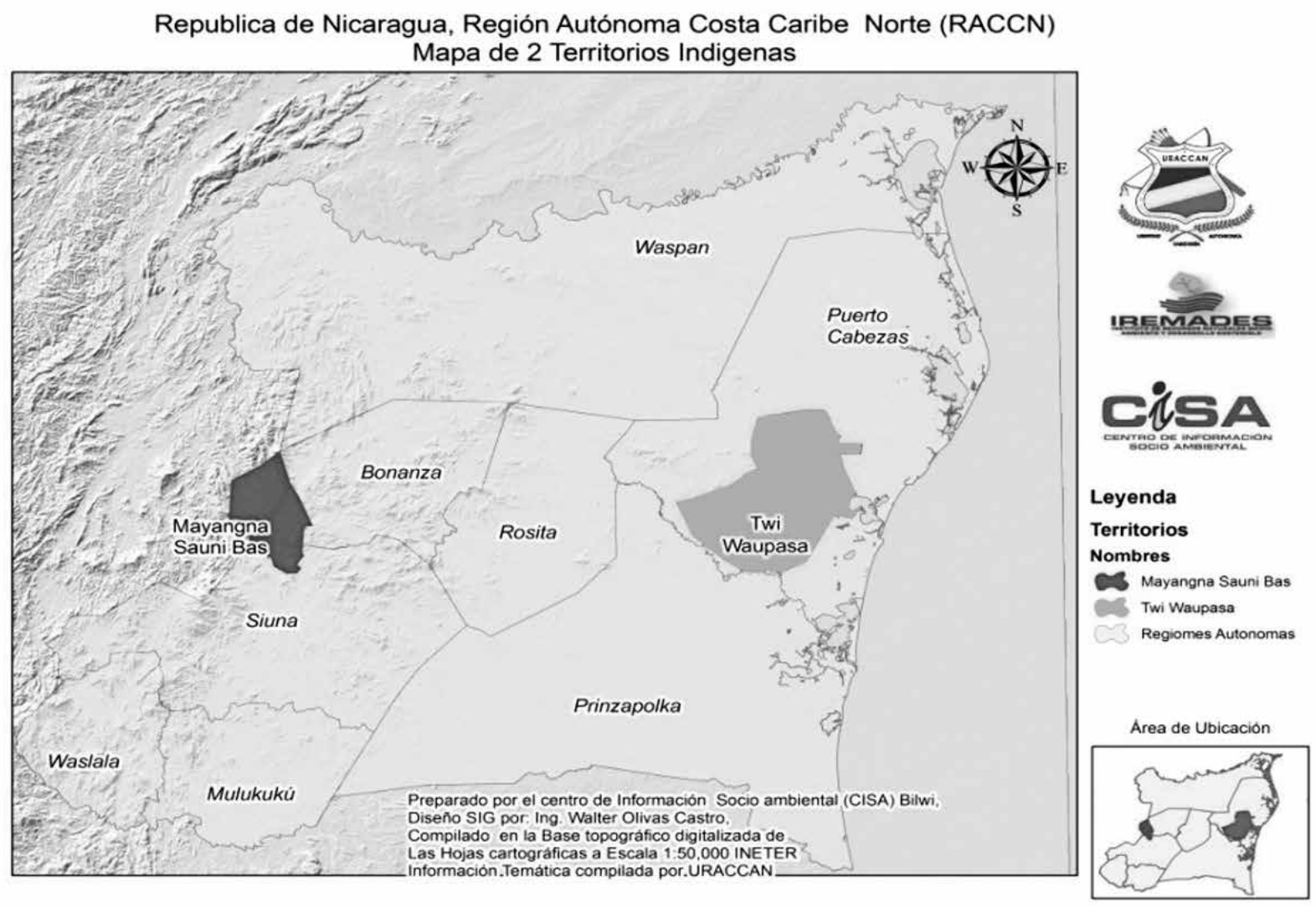

Figura 1. Mapa localización de las áreas de estudio (URACCAN-IREMADES 2015)

El grupo de estudio comprende el conjunto de organizaciones que brindaron un contexto más amplio de los procesos, las cuales son: a) Gobierno central (INETER), b) Gobierno regional (departamento de ordenamiento territorial SEPLAN-GRAAN), c) Gobierno municipal (alcaldías de Puerto Cabezas y Siuna), d) Instituciones y ONGs (GIZ-FISE, universidades), e) Gobiernos territoriales (Twi Waupasa y Mayangna Sauni Bâs)

\section{Resultados y discusiones. 1 Caracterización metodológica de ordenamiento territorial existentes en la Costa Caribe Norte}

\subsubsection{Dinámica territorial y ordenamiento territorial de la Costa Caribe de Nicaragua}

Actualmente las Regiones autónomas tienen una complejidad de gobernanza, que difieren con el resto del país, estructuralmente pueden reflejarse de esta manera:

1. Nicaragua, como nación tiene su estructura de gobernanza, y las Regiones Autónomas "no estamos en una Isla".

2. Las Regiones Autónomas de la Costa Caribe nicaragüense, norte y sur, tienen sus propias estructuras de toma de decisiones (concejales regionales y gobiernos regionales).

3. Las alcaldías, con sus propias estructuras de gobernanza y ejercen sus acciones concretas. 
4. Los territorios indígenas, a la fecha de hoy titulado 22 territorios que existen en la región, una de las estructuras con mayor auge de gobernanza y derechos ancestrales reconocidos a nivel nacional e internacional.

5. Las comunidades, estructura tradicional con muchas historias diferenciadas que forma parte de los gobiernos territoriales.

6. Son respaldas por la ley $28^{5}$ y la $445^{6}$.

7. Respaldadas por Instrumentos jurídicos y administrativos nacionales (Constitución Política de Nicaragua, leyes 40, 261, 278.

8. Instrumentos jurídicos y de referencia internacional, Convenio 169 de la Organización Internacional del Trabajo (OIT), y la Declaración de las Naciones Unidas.

Sin embargo, con todos estos reconocimientos dados por el gobierno de Nicaragua hacia los pueblos tradicionales de la costa caribe nicaragüense, también es una realidad tangible que los recursos naturales están siendo agotados, hay megaproyectos que están entrando en los territorios indígenas sin el Consentimiento Previo Libre e Informado de los pueblos indígenas, se refleja en los territorios problemas sociales comunes, como: colonos en los territorios indígenas de la región, terceros en los territorios, avance de la frontera agrícola en la mayoría de los territorios, Destrucción de la reserva de Bosawas. Compra y venta ilegales de tierras en todos los territorios indígenas.

\subsubsection{Metodología comparativa del INTER y GIZ-Nueva}

En el siguiente cuadro se presenta una caracterización comparativa de las dos metodologías existentes que ha tenido incidencia en la RACCN.

\footnotetext{
5 Estatuto de Autonomía de la Costa Atlántica de Nicaragua.

6 Ley de Régimen de Propiedad Comunal de los Pueblos Indígenas y Comunidades Étnicas de las Regiones Autónomas de la Costa Atlántica de Nicaragua y de los Ríos Coco, Bocay indio y Maíz.
} 
Cuadro 1. Metodología INETER y metodología Nuevo Fise-GIZ.

\begin{tabular}{|c|c|c|}
\hline $\begin{array}{c}\text { Construcción del } \\
\text { ordenamiento, } \\
\text { INETER }\end{array}$ & & culares a las metodologias \\
\hline Momento 1- Preparación & omento 1- Preparación & \multirow[b]{7}{*}{ 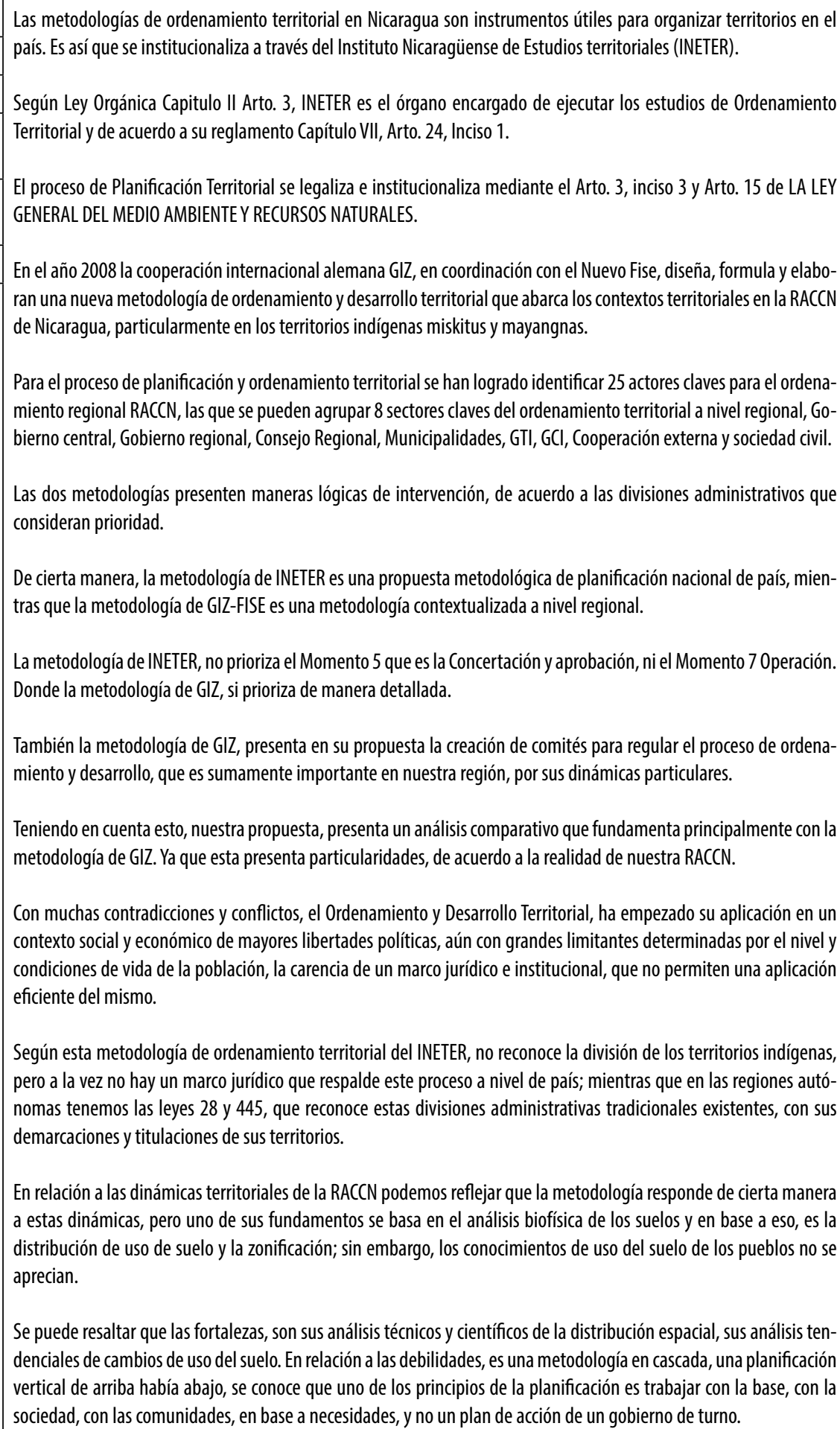 } \\
\hline Momento 2- Diagnóstico & Momento 2-Diagnostico & \\
\hline Momento 3- Prospectiva & Momento 3-Prospectiva & \\
\hline Momento 4- Propuesta & & \\
\hline \multirow[t]{3}{*}{$\begin{array}{l}\text { Momento 5- Instrumen- } \\
\text { tación }\end{array}$} & & \\
\hline & Momento 6-Instrumentación & \\
\hline & Momento 7- Operación & \\
\hline
\end{tabular}


Teniendo esto presente, tanto la metodología de INETER como la metodología de GIZ - Nuevo Fise abarca una dinámica estructural de funcionamiento territorial micro, en base a las realidades de las distribuciones territoriales de la región Costa Caribe del país, teniendo la base las distribuciones territoriales la ley 445 .

Para esto resaltamos que el plan de ordenamiento y desarrollo territorial de Mayangna Sauni Bâs se construyó en base a la metodología propuesta por el GIZ - Nuevo Fise 2010. En el caso del Plan de ordenamiento forestal del territorio de Twi Waupasa se construyó y se diseñó en base a la metodología propuesta por INETER, teniendo referencia el Plan de Ordenamiento Forestal de la RACCN.

Si bien es cierto, y debemos de comprender, que cada una de estas metodologías tiene sus particularidades, pero también sus analogías en sus propuestas metodológicas. Estas analogías, se basa fundamentalmente en sus componentes de Preparación, el Diagnóstico, la fase de la Prospectiva que son similares en cada una de las metodologías; pero también tiene sus diferencias, en relación a las Propuestas, las Concertaciones y Aprobaciones, la Instrumentación y la Operación.

Esto radica principalmente en sus alcances dimensionales como metodologías, ya que la propuesta metodológica de INETER es macro y la metodología propuesta por GIZ/Nuevo Fise es micro, en donde es fundamental la participación de los territorios indígenas miskitu y mayangna en el proceso, para así dar vida y encaminar acciones de desarrollo enmarcados al Plan de ordenamiento y desarrollo territorial. Esto nos lleva a plantear que, el contexto metodológico de INETER en la RACCN, se vuelve muy vulnerable, deficiente y fuera de las realidades de los diferentes contextos sociales, económicos, políticos, culturales y de los pueblos indígenas.

\subsection{Limitantes para los planes de ordenamiento territorial indígena}

\subsubsection{Una estructura vertical para los POT}

Para la elaboración de una propuesta de Metodología de Ordenamiento Territorial para la República de Nicaragua, se ha partido como referencia de la metodología elaborada para los POTEM (Planes de Ordenamiento Territorial Municipal) por INETER.

Quiñonero J. et al (2007), plantea que este modelo se ha utilizado como base de partida para elaborar la presente propuesta destinada al PRONOT, tras su correspondiente adaptación a la realidad nicaragüense en cuanto se refiere a sus características específicas y particulares. De acuerdo a esta metodología plantea que, las estructuras territoriales correspondientes a un nivel determinado no pueden plantearse desde niveles inferiores, por agregación o integración, sino que requieren ser contempladas al nivel territorial que le es propio.

De acuerdo con las reflexiones del punto anterior, generalmente el sistema de ordenamiento territorial previsto en la mayor parte de los países se compone de una serie de instrumentos legales (tipos de planes) de planificación territorial que se suceden en cascada desde los de rango superior a los de rango inferior.

Para mi, la experiencia, lo ideal, es que los planes territoriales alimenten a los planes regionales y despues se deja los planes nacionales porque las realidades las tienen las comunidades y las comunidades son las que saben cuales son sus necesidades.... Dávila J. (Coordinadora CEIMMURACCAN Las Minas)

Esto nos indica que la mayoría de los POT que se han hecho a nivel de la región, responde a un plan macro, priorizando la articulación de los planes macros, obviando a los planes micros, es decir, sin priorizar las necesidades que plantean los territorios. 
Según tal esquema, a cada unidad territorial: país, región, departamento, municipio, se aplica el tipo de figura legal de planificación territorial correspondiente a su nivel; se trata del instrumento más adecuado para prevenir y resolver los problemas y aprovechar las oportunidades correspondientes al rango de la unidad y a prever las estructuras territoriales propias de tal nivel. Porque también, los problemas tienen un rango y se asocian a un nivel de unidad territorial, y les es aplicable el principio de subsidiariedad que señala cómo cada problema debe ser tratado en el nivel administrativo más bajo posible, actuando los niveles superiores solo de forma subsidiaria, es decir, cuando no actúe el nivel inferior (Quiñonero J. et al; 2007).

Este principio de subsidiaridad, no está claro en las divisiones administrativas en la región, y esto refleja Williamson, M. (2016):

Cada administración, no ha asumido responsabilidades adecuadas para dar salida a lo que se plantean en estos planes existentes, y si, hay algunas acciones, porque el plan, responde a programa regional y nacional de gobierno, y esto lo podemos ver en el POF de Twi Waupasa, en donde se ha desarrollado algunas acciones.

En dicho esquema los planes de ordenamiento territorial que se aplican a cada unidad territorial son las referencias y base de partida para la elaboración de los que corresponden a niveles inferiores. De esta forma se garantiza la coherencia o integración vertical de las unidades territoriales de cada nivel, así como las decisiones que se adopten, en las de orden superior, incluyendo las estructuras territoriales que les corresponden. Pero esto no se refleja de esa manera, y se puede ver en algunos grupos focales que se establecieron.

una lideresa de la comunidad de Yulu, expresa que desde sus comunidades han hecho propuestas de desarrollo, que consideran prioridad para las mujeres de su territorio, pero para los actores a nivel municipal y regional, no consideran prioritario, por consiguiente, desarrollar algún tipo de gestión es limitante y expresan que es pérdida de tiempo (Grupo Focal Twi Waupasa)

\subsubsection{Una estructura horizontal integral en los POT}

La metodología para la construcción de POT de la Cooperación Internacional Alemana GIZ y el Nuevo FISE, señala que las comunidades indígenas basan su desarrollo sostenible y humano en el territorio y no en los sectores teniendo en cuenta las condiciones humanas, ecológicas, económicas, sociopolíticas, institucionales, sin perder de vista el peso de la historia y la cultura generada por muchas generaciones anteriores. Donde se recoge el espíritu y el sentimiento de buscar el mejor aprovechamiento de los recursos disponibles y su conexión con el desarrollo del territorio, (GIZ/NUEVO FISE 2011).

No obstante, esto traducido al desarrollo para la construcción de una propuesta para un POT se vuelve desarticulada, ya que para la elaboración y ejecución de las propuestas de los proyectos que surjan en marco del plan deben de responder a un programa o plan nacional, lo cual de cierta manera responderá a los planes políticos de gobierno en turno. A diferencia, que se gestione recursos económicos y humanos desde la cooperación externa; y esto se vuelve mucho más complicado, considerando que en los últimos informes del gobierno (FSLN, 2015-2016), toda la cooperación internacional será canalizada por medio del gobierno central, por consiguiente, al momento de elaborar un POT con las gestiones propias de los gobiernos territoriales es incierta.

Metodológicamente vemos que estos planes de ordenamiento territorial habría que ver si se ajusta a la necesidad de la gente o sea desde el punto de vista científico de análisis está correcto o de variable ahora, realmente se va ajustar al final, no se ha podido demostrar de que, si los ordenamientos territoriales se traducen en beneficio de la gente o realmente contradicen a los intereses de la participación, o en contra más en los pueblos indígenas. Luis Herrera (Coordinador IEPA-URACCAN Siuna). 


\subsubsection{Metodologías y planes desde las ONGs}

De manera general la visión comunitaria y local es que los planes de ordenamiento están alejados de la realidad local. Esto nos lleva a plantear una necesidad urgente de revisar y replantear estos planes, ya que tomando en cuenta esto, las instituciones locales no se han apropiado de la metodología técnicamente, consideran que los actores menos beneficiados de los procesos son los gobiernos territoriales.

Luis Herrera (Coordinador IEPA-URACCAN Siuna), menciona lo siguiente:

Técnicamente la metodología se construyó en consenso, se consultó con varios actores nosotros participamos en esa consulta como universidades, GIZ MARENA, INETER y todas las instituciones, el aparato estatal fueron construyendo ciertos elementos en que tiene sentido científico; el técnico para poder hacer cierta modificación, en el caso de nosotros creemos que esta metodología es muy técnica, muy científica no da el chance de hacer un ordenamiento territorial de manera que la parte humana, el sentido humano se vincule en este la parte humana.

Es necesario que las universidades regionales asuman este tipo de responsabilidad, son las únicas instituciones capaces de poder avanzar cualquier metodología de acuerdo a las realidades de los pueblos. El punto es que las universidades costeñas no han logrado trabajar con los gobiernos territoriales de manera sistemática.

\subsubsection{Una agenda no priorizada por los GTI en el desarrollo de los POT}

El ordenamiento territorial es establecer las áreas de acuerdo al uso que se dan los pueblos indígenas. Como pueblo indígena de forma tradicional tienen ordenadas las áreas de caza, agricultura, pesca, güiresería (minería artesanal) y bosque.

Camaño (2008:70) define que ordenamiento territorial: es el proceso de planeación, evaluación y control, dirigido a identificar y programar actividades humanas compatibles con el uso y manejo de los recursos naturales en el territorio nacional respetando la capacidad de carga del entorno natural para preservar, restaurar el equilibrio ecológico, proteger el ambiente, así como para garantizar el bienestar de la población.

Es necesario hacer primero el plan de ordenamiento territorial, posteriormente las estrategias para el desarrollo sostenible. Esto nos lleva a plantear que estos territorios, el tema de OT no es una prioridad o necesidad latente para ellos, ya que consideran que ellos no deben de hacer un POT de sus territorios, el problema es el mal uso que se ha dado de las empresas nacionales e internaciones que ha entrado en su territorio y han llevado sus recursos, ahora el gobierno nacional plantea hacer un POT de cada uno de sus territorios, y esto se refleja de manera latente en una entrevista a un líder del territorio de Twi Waupasa.

Nuestros ancestros, nuestros indígenas conservan sus recursos naturales y han sobrevivido más de 100 años y viven ahí, sin embargo, no hay ningún ordenamiento territorial y ellos siguen con sus recursos en un ambiente sano, aguas sin contaminación, bosques naturales, fauna silvestre y viven del bosque (Grupo Focal Twi Waupasa).

Esto nos indica la preocupación de actores locales y regionales al plantear esta temática de POT, como refleja Luis Herrera (URACCAN).

Podemos decir que el ordenamiento territorial figura y propone ciertos elementos que se pueden hacer, pero habría que ver si estos se traducen en interés de la gente, este es un plan que se ajusta a las condiciones arqueológicas de los suelos, a las condiciones climáticas, pero no se ha validado si se ajusta a los intereses de la gente. (Luis Herrera, Coordinador IEPA-URACCAN Siuna). 
Así también, en el caso del POT de Mayangna Sauni Bâs, hay una inquietud que se basa en desarrollar POT en un área territorial que posea área protegida, y esto nos lleva a identificar que los desarrollos de estos procesos no están definidos claramente su fin, quizás tenga que ver con interés políticos, intereses gubernamentales, y esto lo indica Zuniga (2008).

Según Zúñiga (2008:50) dice que Ordenar es una acción administrativa del estado, que tiene que ver fundamentalmente con el uso y la ocupación del territorio.

Y esto lo confirma Herrera:

En el caso de los territorios indígenas que están dentro del área de la reserva biosfera de Bosawas son áreas protegidas, no veo sentido de tener un plan de ordenamiento territorial, si Bosawas tiene su plan de manejo, entonces no veo necesario un POT (Luis Herrera, Coordinador IEPAURACCAN Siuna).

\subsubsection{Articulación de instituciones locales gubernamentales, privadas y ONGs}

Se ha podido identificar aproximadamente 25 actores claves para el ordenamiento regional RACCN, las que se pueden agrupar 8 sectores claves del ordenamiento territorial a nivel regional, Gobierno central, Gobierno Regional, Consejo Regional, Municipalidades, GTI, GCI, Cooperación externa y sociedad civil.

No obstante, en los tópicos anteriores se ha visto que de cierta manera la "participación" de las instituciones locales, ONGs, GTI y GCI en los procesos, pero, esto no significa que haya una articulación propia de una agenda común, si no, más bien responde a una necesidad de participar en el proceso y el aprendizaje en la construcción de las metodologías.

Esto tiene mucha relación con el planteamiento de Massiris, (2001), que en la mayoría de los casos a nivel de los países de Latinoamérica, la participación social sólo tiene un carácter consultivo y, por tanto, no incide significativamente en las decisiones finales. Normalmente consiste en usar a unos pocos ciudadanos para tratar de mostrar un plan consensuado y, por lo tanto, legítimo socialmente, contrario a la naturaleza de un verdadero proceso participante, consistente en una negociación social y política del poder sobre el espacio, una construcción colectiva del modelo de uso y ocupación del territorio que se propone como imagen objetivo.

Por consiguiente, se desarrolla el documento base del POT, pero no hay un proceso de empoderamiento de cada uno de estos actores, menos las implementaciones de acciones plasmadas en el documento, y esto se puede apreciar tanto en los dos planes de ordenamiento (Mayangna Sauni Bas y Twi Waupasa).

Esto es muy claro en la lógica de la articulación de los diferentes planes de desarrollo que están surgiendo a nivel de las comunidades, territorios, municipios, región y país. Cada uno de estos sectores está apostando sus acciones que más le corresponda y beneficie a ellos.

El plan de desarrollo humano tiene una lógica, el gobierno central permitió que los gobiernos regionales pudieran construir sus propios planes de desarrollo, pero no hay vinculación, el gobierno municipal tiene cuatro ejes y 15 lineamientos de desarrollo local, entonces cuando nosotros vemos que, los planes de desarrollo comunitario y pueblos indígenas tampoco corresponde a lineamientos municipales, o sea que aquí hay una cosa interesante cada quien tiene su propio plan, la lógica es que fuera un solo plan a nivel nacional, no está el proceso de articulación desde la nacional, regional, municipal, territorial. (Luis Herrera, Coordinador IEPA-URACCAN Siuna).

Este fenómeno negativo de planificación, ha conllevado de cierta manera a considerar que los planes de desarrollo y de ordenamiento se deben desarrollar de manera propia, sea esta comunal, territorial y con sus actores propios, sin la necesidad de la articulación exógena. Pero lo que, si es cierto, esta práctica 
no es la más adecuada, ya que el principio de la planificación y la base fundamental para el desarrollo es la articulación, los roles y los compromisos que sumen cada uno de los actores; no obstante, cada área de gobierno retoma, plasma y desarrollo sus acciones de acuerdo a sus necesidades y demandas que consideren prioridad para sus pueblos.

\subsection{Dificultades para la implementación de los planes de ordenamientos territorial indígenas}

La viabilidad para operativisar y echar andar tu plan depende de qué recurso puedes captar (económico y técnico) y esto normalmente viene del gobierno regional o nacional o talvez de alguna cooperación internacional, que sea de desarrollo u ordenamiento (MarcosWilliamson, Director IREMADES URACCAN 2016).

En todo proceso de construcción de los POT, la implementación es el eje fundamental, ya que es la fase en donde se desarrollarán acciones concretas en marco al plan. Aquí te dirá si el plan construido, tendrá los respaldos necesarios para ser ejecutados por parte de los integrados de la propuesta y la articulación de ella con otras acciones institucionales locales, regionales, nacionales e internacionales.

De acuerdo a Zúniga H. y Lippert T. (2008), señalan que la implementación de los planes de ordenamiento y desarrollo regional con las dinámicas territoriales indígenas, se debe de revisar dos procesos que es importante en la implementación de ella, las cuales son: la instrumentación y la operación.

\section{La instrumentación}

Consiste en la creación de diferentes medios y herramientas (directrices, normas, reglamentos, mecanismos de gestión, planes de inversión), que garantizan la aplicación de manera efectiva de las declaraciones del nuevo POT, Zúniga H. y Lippert T. (2008).

En este caso se debe de tomar en cuenta los siguientes aspectos:

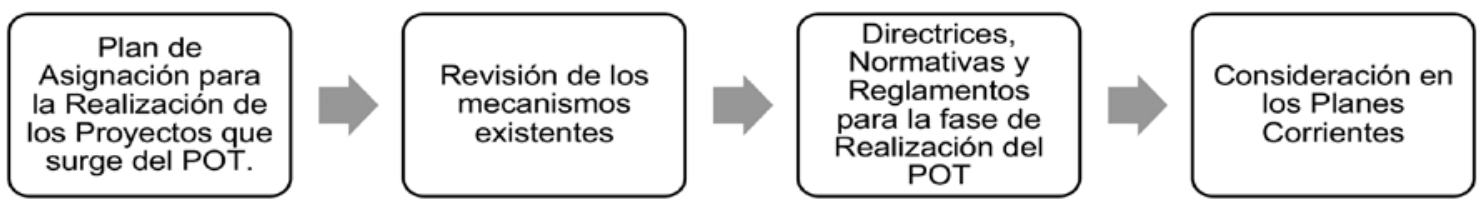

En el grupo focal desarrollado en el territorio de mayangna Sauni bas, han considerado que el PDOT,

"Es un instrumento de gestión, ya que en base a esto han gestionado algunos proyectos de desarrollo territorial, como son la crianza de cerdos, construcción de pozos, producción agrícola, planeación de cacao y cerca viva dentro del territorio (límites territoriales)", Grupo Focal 2 Mayangna Sauni Bas 2015.

En este paso es muy importante clarificar, tanto en los niveles territoriales, municipales, regionales y nacionales, que proyectos, le corresponden asumir, es importante identificar las tareas clásicas en cada nivel de estado.

Zúniga H. y Lippert T. (2008), plantean que para el tema de ordenamiento territorial es obligatorio el principio de subsidiaridad. Cada nivel administrativo tiene sus propias tareas y su soberanía definido, entendiendo que los requerimientos a nivel internacional, tienen que estar conectados con los deseos y visiones nacionales, el nivel regional tiene una visión nacional conectada con las visiones de la región y los municipios tienen una visión regional conectada con la visión de cada municipio. 
El mismo principio de subsidiaridad, obliga a los diferentes niveles administrativos a coordinar según el método de jerarquía.

El territorio indígena tiene sus propias aspiraciones ellos dicen de preservar sus territorios, pero ellos también tienen su visión, quieren vivir bien entonces ellos tienen una propuesta, como yo le digo si nosotros conocíamos el documento del POT podríamos decir la vamos a concatenar con el trabajo, y la idea también con el proyecto que está formulando es incluir todos los territorios, pero como le decíamos lo estamos trabajando (Diala Reyes, Responsable del departamento de planificación Alcaldía Municipal Siuna).

Luego se revisa los mecanismos de gestión existentes a nivel regional, en cuanto a su funcionamiento e integridad. Sobre todo, en la parte de control y evaluación de los proyectos, se debe ver muy claro a quien le corresponde, cual tarea y ver si los mecanismos existentes están capaces de asumir y realizar los proyectos del POT.

Así también, es una gran equivocación considerar que el plan ya está apropiado por los actores beneficiados del proceso, esto ha llevado que en el territorio de Mayangna Sauni Bâs se ha gestionado algunas acciones, pero pocos proyectos enmarcados al plan de ordenamiento como se debería, a largo plazo. En el caso del Plan de ordenamiento forestal del territorio de Twi Waupasa, en el año 2015-2016 se está ejecutando dos proyectos que surgió en marco al POF; esto se debe a que la universidad URACCAN desde el IREMADES-CISA ha brindado acompañamiento continuo a este territorio desde el año 2013, en marco al POF de la RACCN.

Una de las debilidades que te podría decir es "el trabajo de extensión, en URACCAN se hace con los fondos de la cooperación externa, y si no tienes como intervenir en esas comunidades porque terminó la cooperación externa, en este caso siento que una dificultad es que todavía las comunidades no están empoderadas, para poder hacer ellas solas, ir solas a hacer el trabajo o sea, necesitan todavía seguimiento, Dávila J. (Coordinadora CEIMM-URACCAN Las Minas).

Las comunidades no están empoderadas para tomar decisiones para el bienestar de la comunidad. Por eso todavía hay comunidades o territorios que son manipulados por partidos políticos y por las mismas autoridades por sus propios intereses personales. Es decir, siempre esperan a los organismos no gubernamentales.

En la propuesta del PRODT 2008, se plantea la creación del CAS Comité de Auditorio Social. Zúniga H. y Lippert T. (2008), señalan que los Comité de Auditoría Social (CAS), tienen la obligación de formular informes regulares sobre su trabajo. Estos informes se dan a conocer a la población a través de los diarios nacionales y/o publicaciones del Gobierno y Consejo Regional. Así la población tendrá un control directo sobre la realización de los resultados del PRODT.

En caso de los territorios indígenas el que debe de dar salida, a la propuesta del POT son los gobiernos territoriales, con sus estructuras, así como refleja Herrera.

El nivel que es el encargado de darle vida al plan de desarrollo del ordenamiento territorial es el gobierno territorial apoyado de la nación mayangna en el caso de mayangna sauna bâs, ellos son como la estructura, el plan es un organigrama que tiene que administrar a quien le corresponde de hacer, ellos son los que articulan, ejecutan y planifican (Luis Herrera, Coordinador IEPA-URACCAN Siuna).

Para la elaboración del plan de ordenamiento territorial el gobierno territorial está bajo la administración de la nación Mayangna, en el caso del territorio Mayangna Sauni Bas. 
El Consejo Regional, deberá de generar un conjunto de medios y herramientas específicas tales como políticas locales, normativas específicas, mecanismos de gestión, planes de inversión anual y multianual, entre otros, que permitirán la aplicación de los mandatos y orientaciones del nuevo Plan Regional que no están contenidos en la legislación nacional y regional. Se elabora un mapa sobre la distribución territorial de las inversiones en la RACCN.

También se informa del conocimiento a los diferentes niveles administrativos (vecinos), sobre el mapa de distribución de los proyectos, para que ellos puedan considerar las acciones en sus planificaciones.

\section{La operación}

La operación consiste en la ejecución de las acciones propuestas por el POT, con el fin de establecer los escenarios definidos en los pasos anteriores, mediante la ejecución de acciones y proyectos, la activación de los mecanismos de control, seguimiento y evaluación del plan, Zúniga H. y Lippert T. (2008).

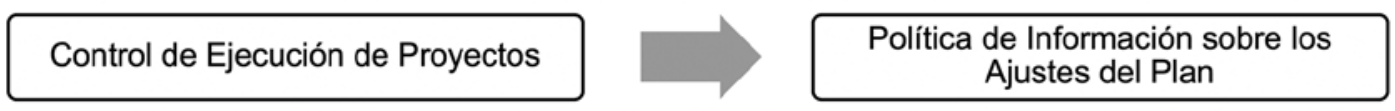

En la región es necesario que el gobierno y consejo regional, busquen apoyo de la cooperación internacional, nacional y empresas privadas, para que puedan financiar, acompañar y complementar de manera sinérgica los programas y proyectos contenidos y que surgen del POT. Pero, es una realidad que tanto los territorios indígenas y el gobierno regional, en su agenda de trabajo y de implementación de acciones "no es prioridad el ordenamiento espacial de sus territorios"; por consiguiente, el escaso presupuesto que entra en estos territorios o a nivel regional, no se usa en estas acciones.

Otro de los fenómenos de administración y gobernanza que sucede al momento de la implementación de los POT, es la falta de articulación por las incidencias de los partidos políticos, y esto lo refleja el territorio Sauni Bas y Twi Waupasa, 2015. "Los partidos políticos que han gobernado fue una dificultad para la coordinación, porque nos decían que no somos del mismo partido de turno, y esto nos cerraba todas las puertas de gestión".

Este malestar también lo refleja el líder territorial de Twi Waupasa (Jorge Mendoza, 2015);

El territorio de Twi Waupasa, en casi su totalidad pertenece al partido YATAMA, y esto nos ha llevado a que los otros niveles administrativos, de referencia tales como el gobierno y la nacional, no, nos ha reconocido nuestra autonomía de gobernanza y nuestras gestiones pertinentes a nuestros procesos de planificación como territorio, ¿Qué hacemos, ellos están de turno?, ha esperar.

Así también, es importante considerar la situación de tenencia de la tierra al momento de la formulación de un POT, así como las lógicas y las articulaciones con las leyes naciones vigentes, como resalta Diala Reyes (Responsable del departamento de planificación Alcaldía Municipal Siuna).

Lo que pienso a lo que dicen las leyes hay una gran diferencia, la ley te limita, el territorio dicen yo quiero tener carretera pero la ley no les permite porque están en una área en la zona núcleo de la reserva biosfera entonces ¿cómo hacer? ya es del territorio, del gobierno municipal no puede hacer mucho, tiene que elevar a otra instancia esa petición entonces el gobierno municipal dice si ustedes consiguen el permiso nosotros llegamos con la carretera hasta donde quieran, en el caso del territorio Mayangna Sauni Bâs.

De acuerdo al plan estratégico regional y municipal no coincide con el plan de ordenamiento territorial de los territorios o que no tiene la misma visión que los territorios y de las comunidades. Es decir, el 
gobierno central y el gobierno regional no permiten que la comunidad sea quien decida sobre los recursos naturales y decidir sobre el desarrollo que ellos quieren. De alguna manera está bien, pero es triste cuando el gobierno si decide sobre esos recursos sin haber consultado y haber tenido aval de la comunidad.

A la hora de construir Plan de Ordenamiento Territorial tiene que nacer desde la visión de los comunitarios, pero muchas veces los organismos llevan el modelo y solo lo rellena por esa razón no coincide con la visión de la comunidad.

Para Zúniga H. y Lippert T. (2008), el Gobierno Regional como el Consejo Regional fundaron y complementaron las herramientas para esta fase con el fin de controlar en conjunto las ejecuciones de los proyectos tanto físico como financiero, según las declaraciones del PRODT de una manera transparente.

\begin{tabular}{|c|c|c|c|}
\hline $\begin{array}{l}\text { Comités de } \\
\text { auditora social } \\
\text { (CAS), } \\
\text { funcionando }\end{array}$ & \multicolumn{2}{|c|}{$\begin{array}{l}\text { Los CAS retroalimenta a las estrategias, } \\
\text { programas y proyectos del Plan Regional de } \\
\text { Ordenamiento y Desarrollo Territorial. A través de } \\
\text { los informes y recomendaciones sobre la } \\
\text { realización de los proyectos }\end{array}$} & $\begin{array}{l}\text { secretarias del Gobierno } \\
\text { Regional, tendrán siempre que } \\
\text { monitorear de manera regular, } \\
\text { que los sectores económicos }\end{array}$ \\
\hline & 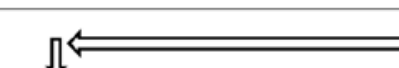 & & ل \\
\hline \multicolumn{2}{|c|}{$\begin{array}{l}\text { GRACCN informará continuamente a la población en } \\
\text { las lenguas usadas en la RACCN sobre los ajustes } \\
\text { realizados en el Plan Regional de Ordenamiento y } \\
\text { Desarrollo Territorial. }\end{array}$} & \multicolumn{2}{|c|}{$\begin{array}{l}\text { Informará de manera oficial a todas las instituciones } \\
\text { estatales, regionales, municipales y territoriales. De } \\
\text { esta misma manera, se involucrará a la sociedad civil } \\
\text { en todo el proceso del avance }\end{array}$} \\
\hline
\end{tabular}

Figura 2. Recomendado para el proceso de implementación a nivel de la RACCN

Con estas mismas dinámicas de comunicación, tanto el Plan de desarrollo y Ordenamiento del territorio de Mayangna Sauni Bâs, como el Plan Ordenamiento Forestal del territorio de Twi Waupasa indican que los responsables en las divulgaciones y dar a conocer sobre los avances de sus planes son la junta del gobierno territorial indígena, con el acompañamiento de la institución, organizaciones que acompañaron en realizar dicho plan.

No obstante, esto dos planes tienen un proceso de seguimiento, monitoreo y evaluación en periodos determinados, en el caso del POF de Twi Waupasa va del 2015 al 2025, en donde se pretende que cada 3 años se haga un proceso de evaluación de los proyectos plasmados, y el cumplimiento de ella. Los responsables de evaluarlo es el GTI con el apoyo de la universidad URACCAN.

\section{Metodología de ordenamiento territorial para los pueblos indígenas del Caribe norte}

La metodología tiene 5 momentos estratégicos, con sus 41 acciones que establecen un parámetro de análisis y de construcción de los planes de ordenamiento territorial.

En la siguiente figura se detalla de manera cronológico el proceso metodológico: 


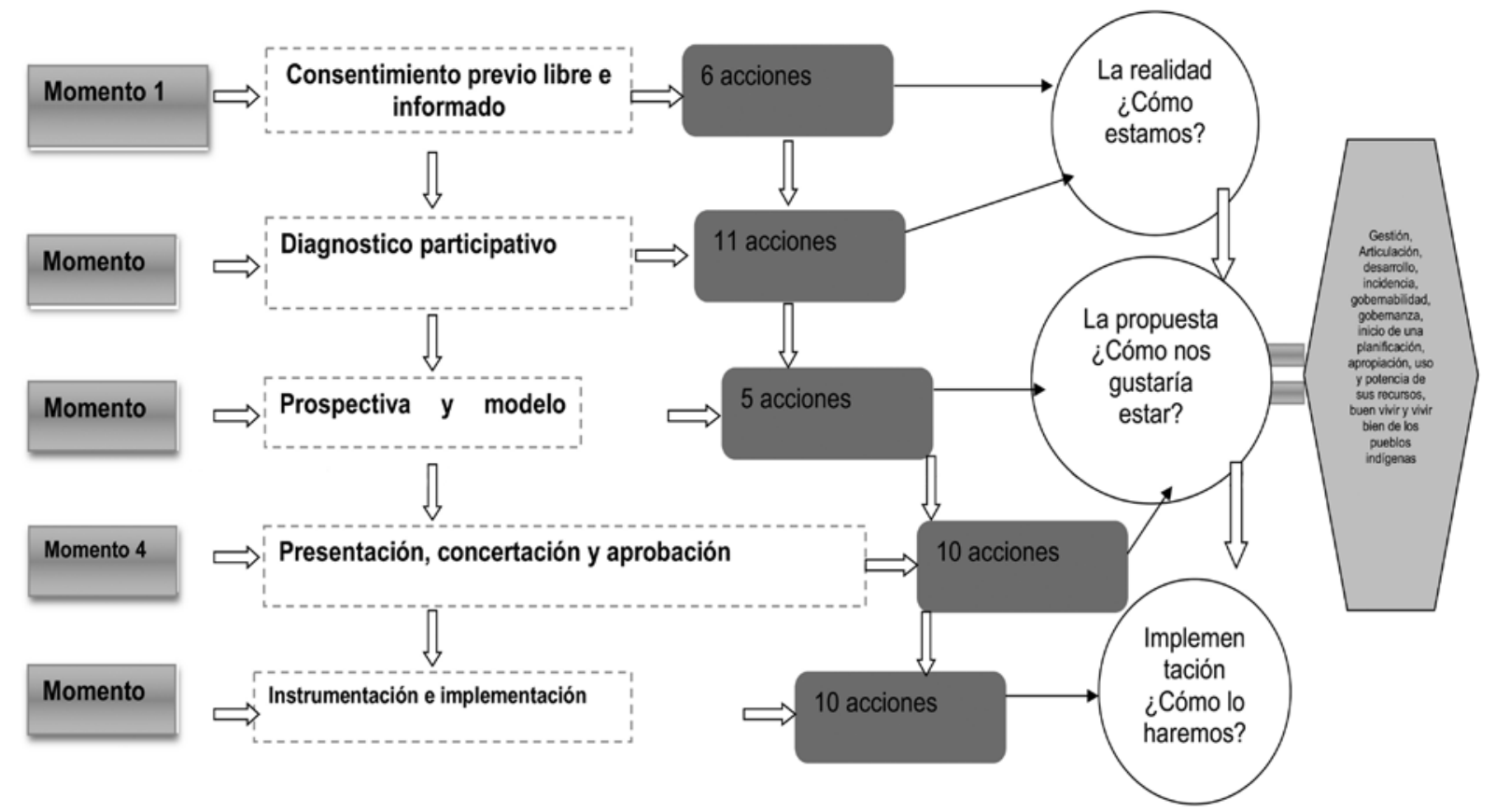

Figura 3. Ruta metodológica de Ordenamiento territorial que responde a la realidad territorial de los pueblos indígenas de la RACCN.

\section{Conclusiones}

1. A nivel nacional y regional los estudios de Ordenamiento y Desarrollo Territorial nacional, tienen su enfoque en las municipalidades.

2. La metodología de INETER no reconoce el contexto de los territorios indígenas de la RACCN, por consiguiente, alcanza o prioriza las divisiones nacional, departamental y municipal.

3. El nuevo FISE-GIZ, establece una metodología más participativa, e integrando algunas particularidades propias de los territorios indígenas, sin embargo, no hay un proceso de empoderamiento de los comunitarios.

4. Los limitantes para la elaboración de los planes de ordenamiento territorial indígenas se basan en cinco brechas: Una estructura vertical para los POT, Una estructura horizontal integral en los POT, Metodologías y Planes construidos por ONGs, Una agenda no priorizada por los GTI en el desarrollo de los POT, Articulación de las instituciones locales gubernamentales, privadas y ONGs.

5. Se desarrolla el documento base del Plan de ordenamiento de sus territorios, pero no hay un proceso de empoderamiento de cada uno de estos actores (tendencias, zonificaciones), menos las implementaciones de acciones plasmadas en el documento, y esto se puede apreciar en los dos planes de ordenamiento (Mayangna Sauni Bas y Twi Waupasa).

6. Los planes de ordenamiento se deben de desarrollarse de manera propia, sea esta comunal, territorial y con sus actores propios, sin la necesidad de la articulación exógena. Pero lo que sí es cierto, esta práctica no es la más adecuada, ya que el principio de la planificación y la base fundamental para el desarrollo es la articulación, los roles y los compromisos que asumen cada uno de los actores; no obstante, cada área de gobierno retoma, plasma y desarrolla sus acciones de acuerdo a sus necesidades, demandas que consideren prioridad para su pueblo y su plan de política partidaria. 
7. Se identifican 25 actores claves para el ordenamiento de los territorios, los que se pueden agrupar en 8 sectores claves del ordenamiento territorial a nivel regional, Gobierno central, Gobierno regional, Consejo Regional, Municipalidades, GTI, GCI, Cooperación externa y sociedad civil.

8. La propuesta metodológica que se propone responde a la realidad territorial de los pueblos indígenas de la Costa Caribe Norte de Nicaragua, tiene 5 momentos estratégicos (Consentimiento previo libre e informado, Diagnóstico participativo, Prospectiva y modelo futuro, Presentación, concertación y aprobación e Instrumentación e implementación) con sus 41 rutas críticas que establecen un parámetro de análisis y de construcción de los planes de ordenamiento territorial propios de los pueblos indígenas.

\section{Lista de referencias}

Camaño Cecilio (2008). Ponencias Centroamericana sobre el proceso de ordenamiento territorial en Honduras. Matagalpa, Nicaragua.

CONADETI (2010). Diagnóstico del territorio Twi Waupasa, RACCN-Nicaragua.

Hooker A. y Zapata Y. (2012). MÓDULO I PROPEDEÚTICO, Fundamentos de la Práctica Intercultural como Eje de Desarrollo con Identidad de los Pueblos. Maestría en planificación y desarrollo regional con identidad; URACCAN - Siuna RACCN, Nicaragua.

Larson. A y Soto (2012). Territorialidad y Gobernanza. Tejiendo retos en los territorios indígenas de la RAAN, NICARAGUA.

Ley No. 28 (2009). Estatuto de Autonomía de las Regiones Autónomas de la Costa Atlántica de Nicaragua y su Reglamento. Recopilación CEJUDHCAN; Nicaragua.

Ley No. 445 (2009). Ley del Régimen de Propiedad Comunal de los Pueblos Indígena y Comunidades Étnicas de las Regiones Autónomas de la Costa Atlántica de Nicaragua y de los Ríos Bocay, Coco, indio y Maíz. Recopilación CEJUDHCAN; Nicaragua.

Massiris A. (2010). Ordenamiento Territorial y Procesos de Construcción Regional. www.banrepcultural.org/ blaavirtual/geografia/masir/1.htm

Massiris A. (2002); Ordenación del Territorio en América Latina; Universidad Pedagógica y Tecnológica de Colombia-UPTC - Departamento de Geografía.

http://www.ub.edu/geocrit/sn/sn-125.htm

Mejía. Javier (2008). Importancia del Ordenamiento Territorial en el Desarrollo Nacional. Instituto Nicaragüense de Estudios Territoriales - INETER; Managua Nicaragua.

Narvaez, K. (2014). Plan de ordenamiento Territorio Twi Waupasa. URACCAN - INAFOR; Bilwi-Nicaragua.

Mendoza-Lewis J. (2009). Diagnóstico-Tasba Raya, enfoque de gobernabilidad comunitaria y territorial. URACCAN-IREMADES; Bilwi-Nicaragua.

NUEVO FISE-GIZ (2011). Cartilla Metodológica para la elaboración de planes estratégicos de ordenamiento territorial en comunidades mayangnas y miskitas de la Región Autónoma del Atlántico Norte, Nicaragua. 
NUEVO FISE-GIZ (2012). Plan desarrollo y ordenamiento territorial del territorio Mayangna Sauni Bas. RACCN - Nicaragua.

Quiñonero J. et al (2007). Metodologías de ordenamientos territoriales. INETER; Managua - Nicaragua.

Romero. R y Lorito A. (2007). Colección de Cartillas (I, II, III, IV y V) que corresponden a las etapas a seguir para elaborar los Planes Municipales de Ordenamiento y Desarrollo Territorial (PMODT). INETER/GTZMASRENACE. Managua, Nicaragua.

Zuniga H. y Lippert T. (2008). Plan regional de Ordenamiento y desarrollo Territorial. GRACCN, RACCN, Nicaragua.

Zúñiga Mendieta, Luis (2008). Ponencias Centroamericana sobre el Proceso de ordenamiento y desarrollo territorial en Nicaragua. Matagalpa, Nicaragua. 